\title{
A novel model of occlusive thrombus formation in mice
}

\author{
Takeshi Sasaki ${ }^{1,2}$, Masafumi Kuzuya ${ }^{1}$, Xian-Wu Cheng ${ }^{1}$, Kae Nakamura ${ }^{1}$, \\ Norika Tamaya-Mori ${ }^{1}$, Keiko Maeda ${ }^{1}$, Shigeru Kanda ${ }^{1}$, Teruhiko Koike ${ }^{1}$, \\ Kohji Sato ${ }^{2}$ and Akihisa Iguchi ${ }^{1}$
}

${ }^{1}$ Department of Geriatrics, Nagoya University Graduate School of Medicine, Nagoya, Japan and ${ }^{2}$ Department of Anatomy and Neuroscience, Hamamatsu University School of Medicine, Shizuoka, Japan

\begin{abstract}
A novel model to induce occlusive thrombus formation was developed in mice in vivo. Mice were simultaneously treated with ligation and cuff placement at the left carotid artery. At 7 days after the treatment, occlusive thrombus was observed at the intracuff region, but not in the distal and proximal regions of the cuff, and not induced by a single treatment of ligation or cuff placement. The plasma levels of von Willebrand factor (vWF), which represent the endothelial status, were significantly increased in combined treatment of ligation and cuff placement 1 day after the operation. Whereas no significant changes in plasma vWF were observed in either single treatment of ligation or cuff placement. The expression of VWF, considered to be the endothelial marker, was detected on the luminal surface distal and proximal to the cuff and the carotid artery in the single treatment groups treated with either ligation or cuff placement, but was not detected in the intracuff region. Furthermore, the binding of Griffolia Simplicifolia Lectin-I (GSL-I) and endothelial nitric oxide synthase (eNOS) expression indicating the endothelial integrity was not detected in the intracuff region. Intermittent injections of ancrod, which decreases the plasma fibrinogen, inhibited occlusive thrombus formation in the intracuff region. The expression of eNOS was detected at the distal and proximal but not the intracuff region of the carotid artery treated with ancrod. Daily administration of aspirin significantly suppressed the thrombus formation in this model. These results indicate that occlusive thrombus formation accompanied by endothelial damage or dysfunction is induced by the combined application of ligation and cuff placement at the carotid artery, and suggest that this endothelial damage or dysfunction may be one pathogenesis of thrombogenesis in this model.
\end{abstract}

Laboratory Investigation (2004) 84, 1526-1532, advance online publication, 30 August 2004; doi:10.1038/labinvest.3700171

Keywords: cuff; endothelial damage; ligation; mouse; thrombosis

Thrombus formation is a crucial event in several ischemic vascular diseases such as myocardial and cerebral infarctions. Myocardial infarction in particular is mainly caused by occlusive thrombus formation subsequent to the disruption of the atherosclerotic plaque or erosion of the endothelium of coronary arteries. ${ }^{1}$ Since the endothelium suppresses the thrombosis by means of antithrombotic substances such as tissue plasminogen activator, prostacyclin, thrombomodulin and heparan sulfate, ${ }^{2}$ the thrombogenesis is closely linked to the injury and dysfunction of the vascular endothelial cells. Plaque disruption or erosion of the endothelial surface exposes luminal blood to collagen and tissue

Correspondence: Dr M Kuzuya, MD, PhD, Department of Geriatrics, Nagoya University Graduate School of Medicine, 65 Tsuruma-cho, Showa-ku, Nagoya, Japan.

E-mail: kuzuya@med.nagoya-u.ac.jp

Received 6 May 2004; revised and accepted 11 July 2004; published online 30 August 2004 factor, thereby stimulating thrombus formation through the activation of the platelet and coagulation cascade. ${ }^{1}$

Pathological animal models are valuable because they are likely to represent human diseases well and complement the insufficiency in cell culture experiments. Mice are appropriate laboratory animals because of their small size, which makes them easy to manage and feed, and their superior potential for reproduction. Furthermore, recent methods of analysis and alterations of genes have enlarged their potential as model animals. However, their small size might sometimes make the experimental operations difficult, for example, injuring the endothelium leading to thrombosis or neointima formation. Therefore, easier and simpler methods that induce thrombosis by means of endothelial injury are required.

Several models of murine thrombosis by vascular injury have been reported using mechanical, ${ }^{3}$ electrical, ${ }^{4} \mathrm{FeCl} 3,{ }^{5}$ photochemical ${ }^{6}$ and laser-induced injuries. ${ }^{7,8}$ The development of thrombi induced by 
most of these methods are very acute, occurring from within a few seconds to $1 \mathrm{~h}$. However, a model in which occlusive thrombus is formed gradually in the carotid artery has not been reported.

In the present study, we report the simple murine model of arterial occlusive thrombosis, which is formed gradually within 7 days. The new model has been developed by combination treatments of murine carotid artery ligation and perivascular cuff placement on the carotid artery, both of which are well-known to evoke intimal hyperplasia. ${ }^{9-11}$ In addition, we examined the utility of this model for evaluation of antithrombotic agents.

\section{Materials and methods}

\section{Mice}

Male mice (C57BL/6J, Japan SLC, Hamamatsu, Japan) used in this study were between 8 and 10 weeks old, and weighed between 22 and $26 \mathrm{~g}$. Mice were provided with a standard diet (Oriental Yeast, Tokyo, Japan) and tap water ad libitum throughout the experimental period. All animal experiments were performed in accordance with the guidelines on animal care of Nagoya University Graduate School of Medicine.

\section{Animal Surgery}

The animals were anesthetized with an intraperitoneal (i.p.) injection of pentobarbital sodium $(50 \mathrm{mg} /$ kg, Dainippon Pharmaceutical, Tokyo, Japan). We used the combined technique of carotid ligation and polyethylene cuff placement (length $2 \mathrm{~mm}$, inside and outside diameter 0.580 and $0.965 \mathrm{~mm}$, respectively, Becton Dickinson, Sparks, MD, USA). This inside diameter was nonconstrictive for the common carotid artery. ${ }^{12}$ Common carotid artery ligation and/ or cuff placement were carried out according to previous reports, ${ }^{10,12}$ and the operating diagram is illustrated in Figure 1a. Briefly, the left common carotid artery was dissected from the surrounding connective tissue and ligated just proximal to its bifurcation with 5-0 silk ligature. The nonocclusive cuff was then placed at $2 \mathrm{~mm}$ proximal to the ligated site and was tied circumferentially with a silk ligature. In control group, the dissection of the left
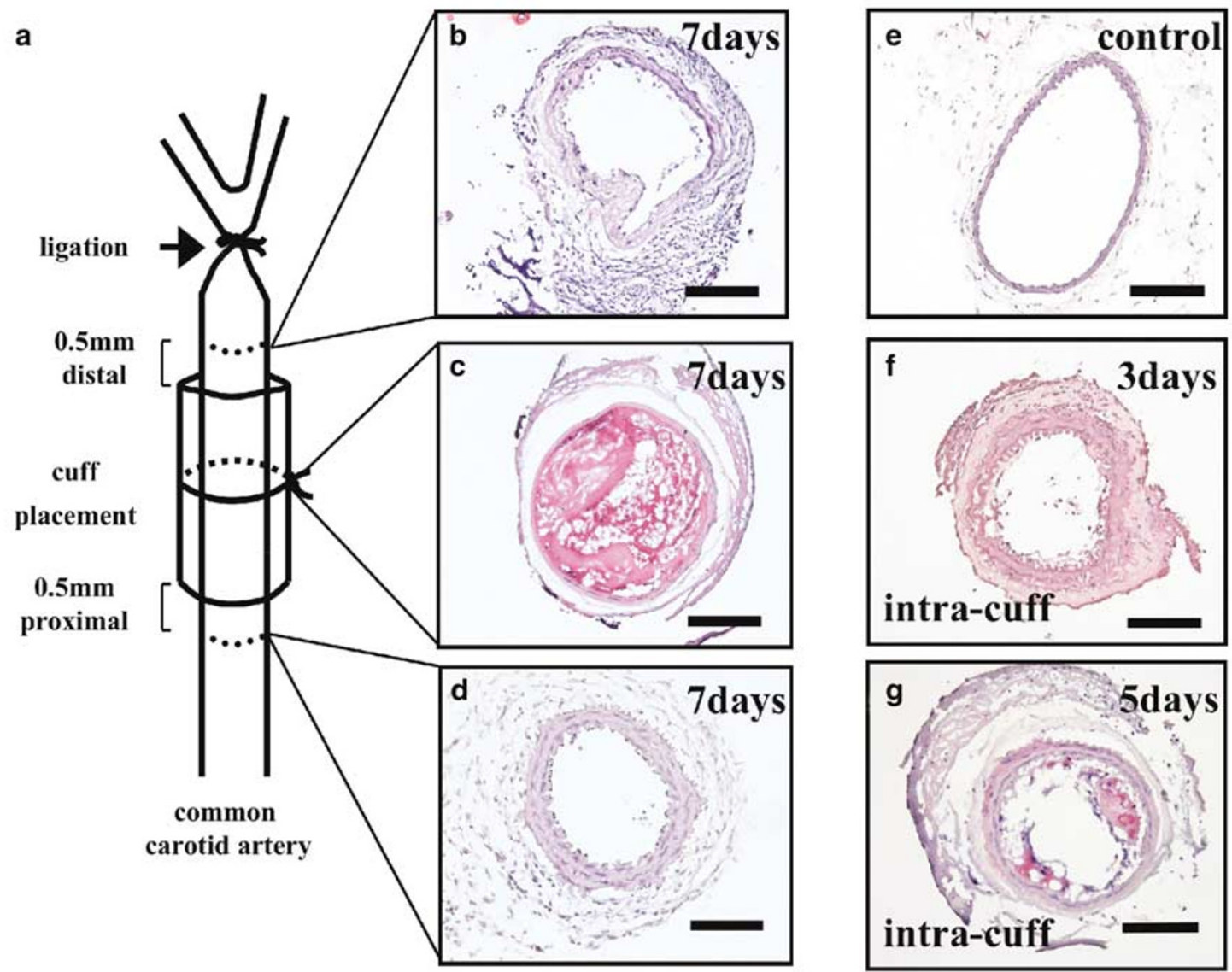

Figure 1 Thrombosis in the carotid artery of ligation-cuff-treated mice. Schematic diagram of ligation and cuff placement (a), and H\&E staining of cross-sections at $0.5 \mathrm{~mm}$ distal to the cuff (b), the center of the intracuff region (c), and $0.5 \mathrm{~mm}$ proximal to the cuff (d). Contralateral carotid arteries (e) at 7 days after the operation, and 3 (f) and 5 (g) days after the operation at the center of the intracuff area. Bars $=100 \mu \mathrm{m}$. 
common carotid artery from the surrounding connective tissue was performed without ligation and/ or cuff placement.

\section{Tissue Collection and Processing}

At various time points after the ligation and cuff treatment (3, 5 and 7 days after surgery; $n=10,9$ and 9, respectively), mice were anesthetized by i.p. pentobarbital injection. Blood samples of mice were collected in heparinized syringes just before perfusion with isotonic saline from the left cardiac ventricle, and mice were perfused through the left cardiac ventricle with isotonic saline and $4 \%$ paraformaldehyde in $0.01 \mathrm{M}$ phosphate buffer $(\mathrm{pH}$ 7.4) under physiological pressure. The carotid arteries were removed and immersed in fixative for $6 \mathrm{~h}\left(4^{\circ} \mathrm{C}\right)$. These arteries were separated into three parts, namely the distal, internal and proximal regions of the collar, after the removal of the cuff. Each vessel was embedded in OCT compound (Sakura Finetechnical, Tokyo, Japan), snap-frozen in liquid nitrogen, and stored at $-20^{\circ} \mathrm{C}$ until use.

\section{Ancrod or Aspirin Administration in Mice}

At 1, 3 and 5 days after the combined application of ligation and cuff placement, the mice were given ancrod (1.5 U/150 $\mu$ l, i.p., Sigma, St Louis, MO, USA, $n=6$ ), and the carotid arteries were collected at 7 days after the operation and stored as described above.

From 2 days before the ligation and cuff placement, aspirin was administrated orally $(0,25$ or $100 \mathrm{mg} / \mathrm{kg} ; n=9,9$ or 11 , respectively, once-daily, Sigma, St Louis, MO, USA) by gastric gavage throughout the experimental period, and the carotid arteries were collected at 7 days after the operation and stored as described above.

\section{Measurements of Plasma vWF}

Plasma was isolated from the blood by centrifugation at $3000 \mathrm{~g}$ for $15 \mathrm{~min}$ at $4^{\circ} \mathrm{C}$ and stored at $-80^{\circ} \mathrm{C}$ until the determination of vWF. Plasma vWF was determined using the latex immuno-assay kit (Roche Diagnostics, Mannheim, Germany) according to the manufacturer's instructions. The data were expressed as percentages relative to sham-operated mice as the control.

\section{Histological, Immunohistochemical and Morphometric Analysis}

Cross cryosections $(6 \mu \mathrm{m})$ were prepared from the carotid artery and stained routinely with hematoxylin and eosin (H\&E). By means of H\&E staining, the existence of the thrombus was examined, and the thrombi were classified into three grades (0: no thrombus, + : unocclusive thrombus, and ++ : occlusive thrombus) according to the occupancy of the lumen.

The corresponding sections on separate slides were used for the Griffolia Simplicifolia Lectin-I (GSL-I) staining and immunohistochemical staining to evaluate the endothelial integrity. The GSL-1 binds specifically to galactosyl residues present on vascular endothelium in mice. ${ }^{13,14}$ The sections were incubated with biotinylated GSL-I $(10 \mu \mathrm{g} / \mathrm{ml}$, Vector Laboratories, Burlingame, CA, USA) for $2 \mathrm{~h}$ at $4^{\circ} \mathrm{C}$. Immunohistochemical staining for $\mathrm{vWF}$ and endothelial nitric oxide synthase (eNOS) were performed with polyclonal vWF (1:200, Dako, Hamburg, Germany) and eNOS (1:250, Transduction Laboratories, Lexington, KY, USA) antibodies and biotinylated second antibody (1:200, goat anti-rabbit IgG, Vector Laboratories). The GSL-I, vWF and eNOS staining were visualized using an ABC-AP kit and Vector Red (all from Vector Laboratories) according to the manufacturer's instructions. Levamisole (Vector Laboratories) was used as the inhibitor of endogenous alkaline phosphatase.

In the experiment of aspirin administration, three sections $(0.5,1.0$ and $1.5 \mathrm{~mm})$ at the intracuff region of each carotid artery were used for morphometric analysis. The inhibitory effect of aspirin on the thrombus formation was evaluated by three sectional average ratio of the thrombus area to lumen area (T/L ratio), and schematic diagram is illustrated in Figure 5a. The measurements of the crosssectional thrombus and lumen area were carried out by using the Scion Image Analysis software (Scion Co., Frederick, MA, USA).

\section{Statistical Analysis}

Data were represented as means \pm s.d. One- or twoway analysis of variance followed by post hoc testing (Scheffe's test) was used for statistical analysis where appropriate at $P<0.05$.

\section{Results}

\section{Thrombogenesis in the Carotid Artery}

The occlusive thrombi were observed at the center of the intracuff region in all carotid arteries at 7 days after the combined application of ligation and cuff placement (Figure 1c). In contrast, no thrombus was seen at $0.5 \mathrm{~mm}$ distal and proximal to the collar (Figure $1 \mathrm{~b}$ and $\mathrm{d}$, respectively). In all animals, no thrombus was present in the contralateral carotid artery as a control (Figure 1e). At 3, 5 and 7 days after operation, the occlusive thrombi were observed in $10.0,55.6$ and $100.0 \%$ of the intracuff region, respectively (Table 1). Nonocculusive thrombi were detected in 40.0 and $33.3 \%$ at 3 and 5 days, respectively (Figure 1f,g and Table 1). A single treatment of either ligation or cuff placement 
produced small thrombi only at the vicinity of the ligation site or no thrombi at all in the carotid arteries, respectively, at 7 days after the operation (Figure 2g and $\mathrm{h}$ ).

\section{Measurements of vWF}

No significant changes in the plasma levels of vWF were detected at 1 and 3 days after a single treatment of ligation (Figure 3). Although vWF levels increased at 1 and 3 days after cuff placement, there were no significant differences compared to the

Table 1 Changes in proportion to thrombus formation in ligation-cuff-treated carotid arteries at the center of the intracuff region

\begin{tabular}{lccr}
\hline \multirow{2}{*}{ Thrombus } & \multicolumn{3}{c}{ Days after the treatments (numbers) } \\
\cline { 2 - 4 } & 3 days $(\mathrm{n}=10)$ & 5 days $(\mathrm{n}=9)$ & 7 days $(\mathrm{n}=9)$ \\
\hline 0 & $50.0 \%(5)$ & $11.1 \%(1)$ & $0.0 \%(0)$ \\
+ & $40.0 \%(4)$ & $33.3 \%(3)$ & $0.0 \%(0)$ \\
++ & $10.0 \%(1)$ & $55.6 \%(5)$ & $100.0 \%(9)$ \\
\hline
\end{tabular}

Classification of the thrombi into three grades (0: no thrombus, +: unocclusive thrombus, and ++: occlusive thrombus) according to its occupancy to the lumen. control ( $P=0.930$ and 0.098 , respectively). Following the combined application of ligation and cuff placement, the plasma vWF dramatically increased at 1 day $(P<0.001)$, and then decreased at 3 days after the operation. At 1 day after the operation, the plasma vWF levels of the ligation-cuff application were significantly higher than both the levels of ligation and cuff placement $(P=0.003$ and 0.021 , respectively).

\section{Lectin and Immunohistochemical Staining}

Endothelial integrity on the luminal surface was verified by staining for vWF and eNOS, and GSL-I binding at various sites of the carotid artery. At 7 days after the operation, the expression of $\mathrm{vWF}$ was observed circumferentially throughout the right common carotid artery as a control (Figure 2d). In carotid arteries with the combined application of ligation and cuff placement, vWF was detected on the inner surface of the vessel wall in extracollar but not intracollar regions in which occlusive thrombus was observed (Figure 2a-c). There was no staining for eNOS or GSL-1 binding at the luminal surface of the intracollar region (Figure $2 \mathrm{e}$ and f). In ligated mice without cuff placement, the expression of $\mathrm{vWF}$ was detected circumferentially on the
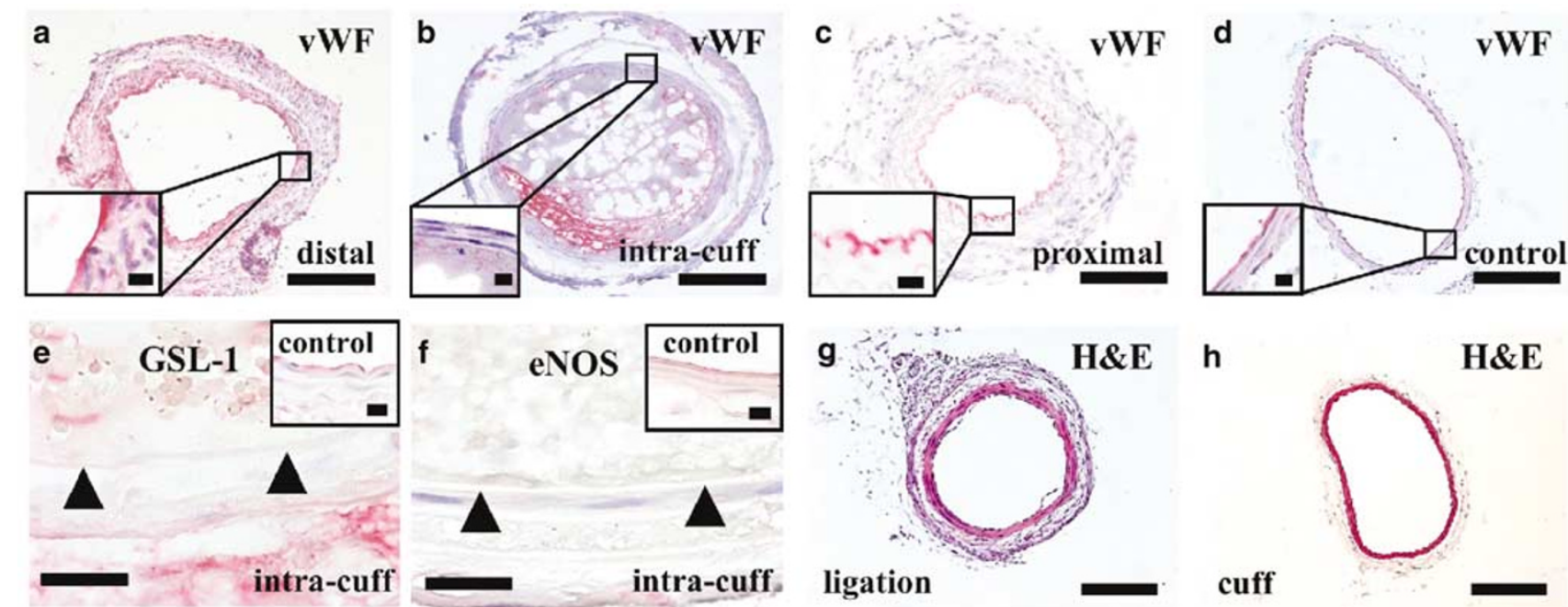

H\&E
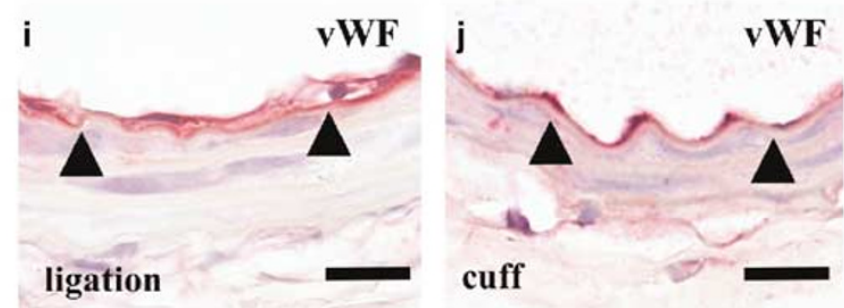

Figure 2 Expression of vWF in ligation-cuff-treated carotid arteries at $0.5 \mathrm{~mm}$ distal to the cuff (a), the center of the intracuff region (b), $0.5 \mathrm{~mm}$ proximal to the cuff (c), and at the contralateral carotid arteries (d) 7 days after the operation. The inserted panels are high magnification views (lower left inset of (a)-(d)). Lectin staining (e) and eNOS (f) expression at the intracuff region of the carotid artery 7 days after the operation, and contralateral carotid arteries as a positive control (upper right inset of (e) and (f), respectively). H\&E staining $(\mathbf{g}$ and $\mathbf{h}$ ) and vWF (i and $\mathbf{j}$ ) expression of the carotid artery at 7 days after a single treatment of ligation ( $\mathbf{g}$ and $\mathbf{i}$ ) or cuff placement (h and j). Arrows indicate internal elastic lamina. Bars $=100 \mu \mathrm{m}$ (a-d, $\mathbf{g}$ and $\mathbf{h})$ and $20 \mu \mathrm{m}(\mathbf{e}, \mathbf{f}, \mathbf{i}, \mathbf{j}$ and figures in the insets). 
luminal surface at the equivalent site of the cuffed region of the carotid artery (Figure 2i). The vWF expression was also detected circumferentially throughout the luminal surface in the intracuff region in the carotid artery with a single treatment of cuff placement (Figure 2j).

\section{Effect of Ancrod on Thrombus Formation and eNOS Expression}

The administration of ancrod blocked the thrombus formation at 7 days in all regions of the ligation-cuff placement carotid artery as well as in the control right carotid artery (Figure 4a-d). The eNOS expres-

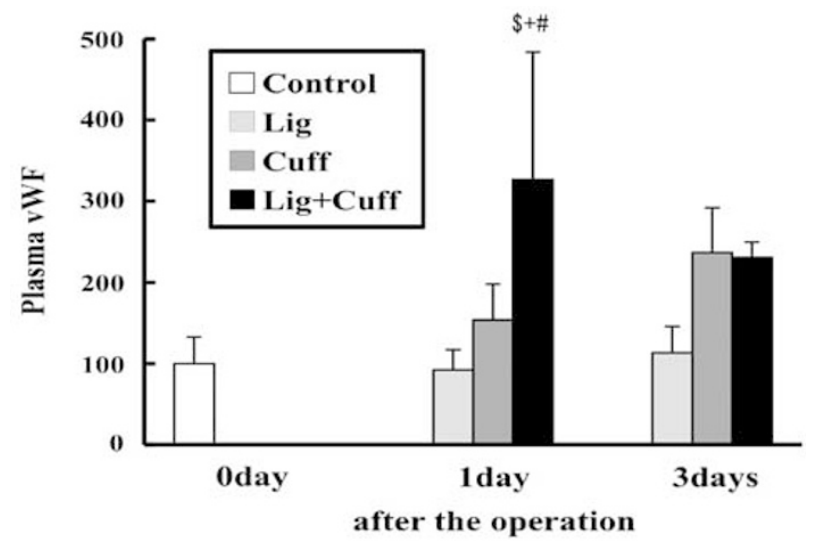

Figure 3 The changes in plasma vWF after various treatments (ligation: Lig, cuff placement: Cuff, and ligation-cuff placement: Lig + Cuff). Sham operation (0 day) as a control group. ${ }^{\$} P<0.05$ vs control. ${ }^{+} P<0.05$ and ${ }^{\#} P<0.05$ vs Lig and Cuff at 1 day after the operation, respectively. sions on the luminal surface were identified throughout the right and left carotid arteries, except in the intracollar region (Figure $4 \mathrm{e}-\mathrm{h}$ ).

\section{Effect of Aspirin on Thrombus Formation}

At 7 days after ligation-cuff treatment, the oral administration of aspirin suppressed the average $\mathrm{T} /$ $\mathrm{L}$ ration compared with that of control (Figure 5a). This inhibitory effect was dose-dependent manner. The $\mathrm{T} / \mathrm{L}$ ratio in $100 \mathrm{mg}$ aspirin-treated group was significantly lower than that in the control group $(P=0.014$; Figure 5b).

\section{Discussion}

The present study clearly demonstrated that the simultaneous treatments of ligation and cuff placement in the mouse carotid artery induced occlusive thrombus formation. In this experiment, the occlusive thrombus was observed at 5-7 days after the treatments. It is known that thrombus formation is prevented by the antithrombogenic function of endothelium and that the disruption of endothelial integrity is one of the pathogenesis of thrombus formation. ${ }^{4,6,7,15}$ We showed that the GSL-1 binding and expression of vWF and eNOS as histological makers of endothelial cells were markedly decreased in endothelium that was accompanied by thrombus formation in ligation-cuff-treated arteries. Thus, the combination of ligation and cuff placement induced the damage of endothelia, which led to the exposure of the subendothelial matrix. The formation of the occlusive thrombus was localized
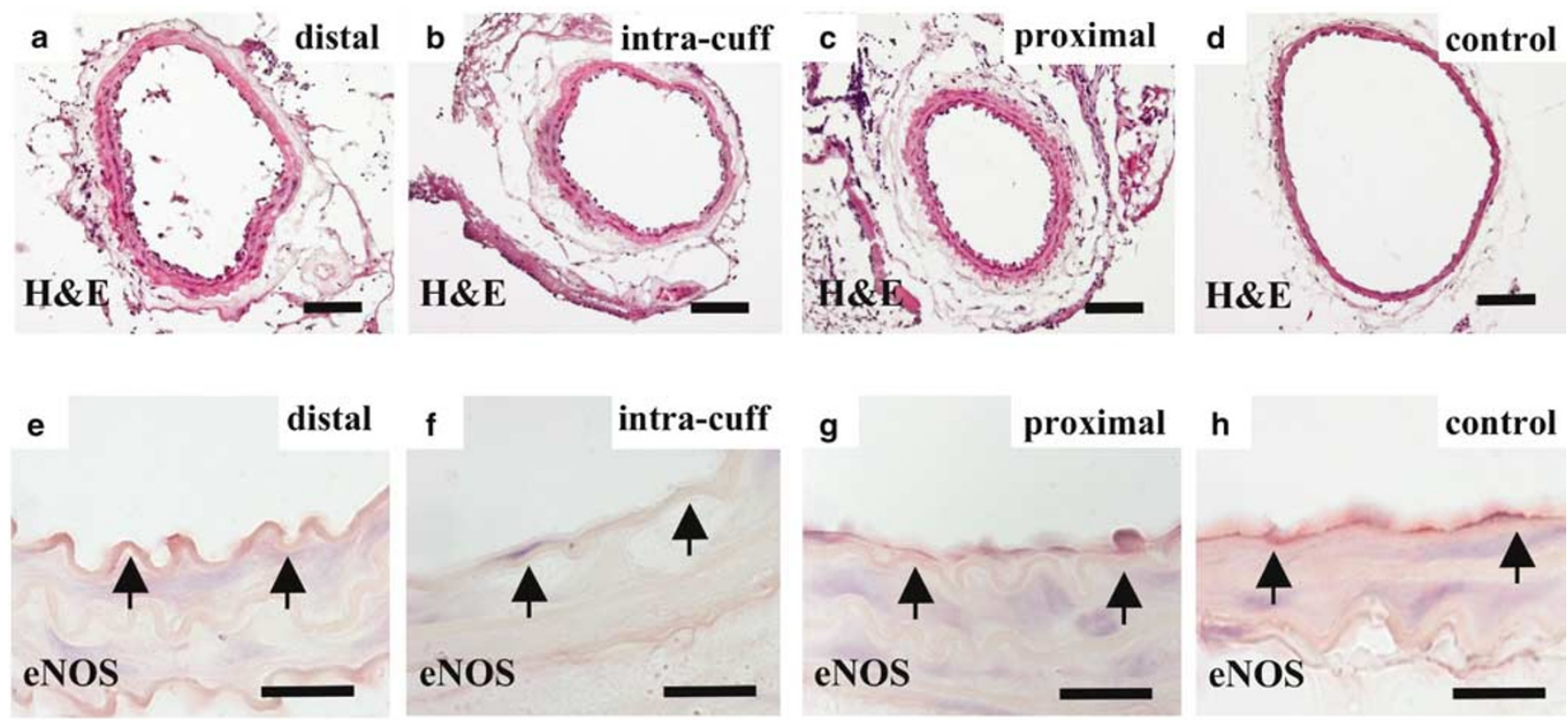

Figure 4 The effect of ancrod injection on thrombus formation in a carotid artery with ligation-cuff treatment. H\&E staining (a-d) and eNOS (e-h) expression. Carotid artery at $0.5 \mathrm{~mm}$ distal to the collar (a and $\mathbf{e}$ ), the center of the intracuff area (b and $\mathbf{f}$ ), $0.5 \mathrm{~mm}$ proximal to the collar ( $\mathbf{c}$ and $\mathbf{g}$ ), and the contralateral carotid artery as the control (d and $\mathbf{h}$ ) at 7 days after the operation. Arrows indicate internal elastic lamina. Bars $=100 \mu \mathrm{m}(\mathbf{a}-\mathbf{d})$ and $20 \mu \mathrm{m}(\mathbf{e}-\mathbf{h})$. 
a

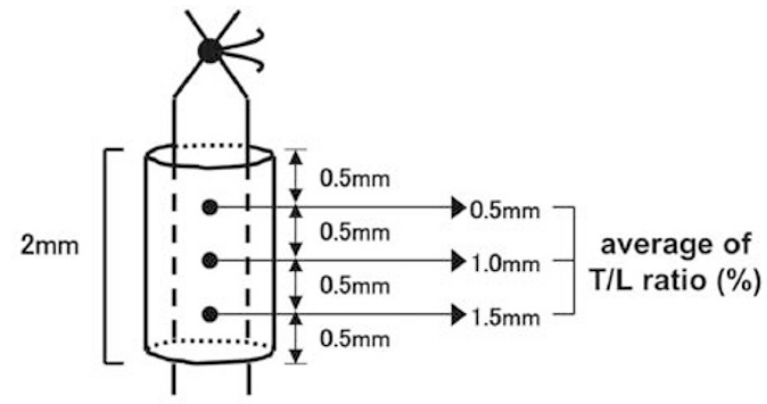

b

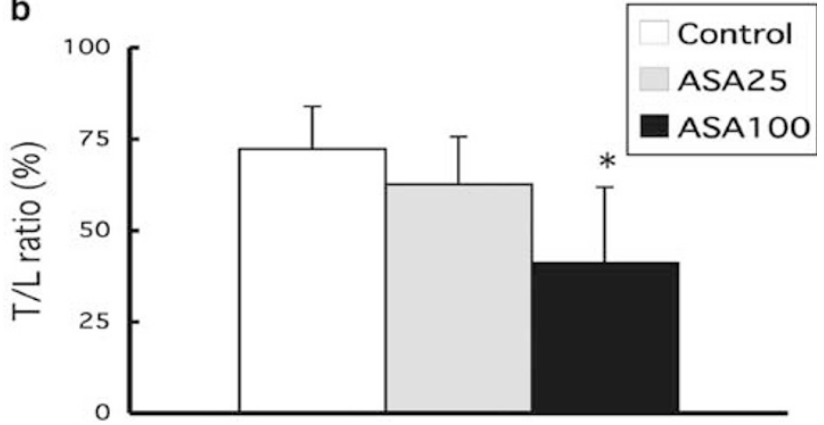

Figure 5 The effect of aspirin administration on the thrombus formation in a carotid artery with ligation-cuff treatment. Schematic diagram of the three points at intracuff region for estimating thrombus area/lumen area (T/L) ratio (a). T/L ratio in vehicle (Control, $n=9$ ), $25 \mathrm{mg}$ (ASA25, $n=9$ ) and $100 \mathrm{mg}$ (ASA100, $n=11$ ) $/ \mathrm{kg}$ body weight per day aspirin treated animals (b). ${ }^{*} P<0.05$ vs control.

in the intracollar region of the carotid artery. This site-specific thrombogenesis seems to be due to the limited damage site of the endothelium of the carotid artery. Indeed, we clearly showed that a dramatic reduction of the histological markers of endothelium, vWF and eNOS expression and GSL-1 binding, was detected at the site-specific intracuff region of ligation-cuff carotid arteries. Single manipulation of a carotid artery ligation as well as cuff placement did not produce an occlusive thrombus in the carotid arteries. In addition, vWF expression remained in the endothelium of arteries that were either ligated or cuffed. Thus, it is suggested that the cuff treatment in addition to ligation caused greater injury to the endothelium at the intracuff region, accordingly, the occlusive thrombus formation was induced at the intracollar region site-specifically. However, it is not clear the cause for the endothelial aberration and occlusive thrombus formation at the intracollar region in the case of simultaneous treatments of ligation and cuff placement. The ligation of carotid artery induces the hemostasis, hypoxia and microthrombi at the proximal region, ${ }^{16}$ and cuff placement provoked inflammation around cuff region. ${ }^{17}$ Therefore, the accumulations of these pathophysiological conditions may participate in the site-specific damage of endothelium and thrombus formation.

Several investigators have proposed the measurement of plasma vWF as an index of endothelial damage in vascular disease. ${ }^{18-21}$ In this study, plasma levels of vWF were significantly elevated at 1 day after the ligation-cuff treatments of the carotid artery, suggesting that the endothelium had been injured by the combined treatment of ligation and cuff placement. The majority of plasma vWF is derived from the endothelial cells, and the elevation of plasma vWF may reflect the leak of vWF from the damaged endothelial cell membrane. ${ }^{21}$ However, since vWF is also contained in platelets, and in pathological conditions characterized by the occurrence of platelet activation, vWF may be released from platelets and may contribute significantly to plasma levels unlike under physiological conditions. ${ }^{22}$ Thus, plasma levels of vWF could not necessarily reflect the actual degree of endothelial cell damage. In this experiment, however, the plasma vWF levels dramatically increased at 1 day after the ligation-cuff treatments before the thrombus observation, and no histological evidence of platelet activation such as platelet aggregation and platelet adhesion to the endothelium was detected in the intracuff region of the carotid artery 1 day after the operation. In addition, a single treatment of ligation did not significantly increase plasma vWF at 3 days after the treatment in the present study, at which activated platelets were observed in proximal to the ligated site of carotid artery. ${ }^{16}$ The contribution to circulating vWF from the platelet is barely significant. ${ }^{23}$ Therefore, it is suggested that platelet activation is not involved in the increase of plasma $\mathrm{vWF}$, at least in the early phase.

The possibility remains that the downregulation of endothelial markers accompanied by thrombus formation might be due to the endothelial injury induced secondarily by the thrombus rather than primarily by the ligation-cuff stimulation. We demonstrated that the intermittent bolus injections of ancrod, which suppresses the thrombogenesis via a reduction of plasma fibrinogen, ${ }^{24}$ inhibited the thrombosis but maintained the decline eNOS expression on the luminal surface at the intracollar region of the ligation-cuff-treated carotid artery. These results led us to conclude that the combination of ligation and cuff placement initially induces endothelial damage, which is subsequently followed by thrombus formation.

In most mouse models of thrombus formation, thrombi were rapidly generated from within a few seconds to less than $1 \mathrm{~h} .{ }^{4-6,25,26}$ These models by means of acute and dramatic injury of endothelium appear not to be suitable for investigating the course and mechanism of endothelium damage or injury, since it is too acute to detect the pathophysiological changes of endothelium after stimuli. This model is likely to be of particular value in studying the relationship between thrombosis formation and the alteration of endothelial cell morphology and function in detail. An endothelial site-specific disruption induced by the combination of ligation and cuff placement provides a unique model for spontaneous 
thrombosis secondary to endothelial cell damages. Therefore, this model provides insight into the physiological role and importance of endothelial function in vivo and constitutes a valuable animal model for the evaluation of new therapeutic strategies for the treatment of thrombogenesis. Indeed, inhibitory effect of aspirin on the thrombus formation was confirmed by using this model. We hope that this thrombus formation model will be utilized in the assessment of various already-known and as-yet-unknown antithrombotic agents in the future.

\section{References}

1 Zaman AG, Helft G, Worthley SG, et al. The role of plaque rupture and thrombosis in coronary artery disease. Atherosclerosis 2000;149:251-266.

2 Jaffe EA. Cell biology of endothelial cells. Hum Pathol 1987;18:234-239.

3 Stockmans F, Deckmyn H, Gruwez J, et al. Continuous quantitative monitoring of mural, platelet-dependent, thrombus kinetics in the crushed rat femoral vein. Thromb Haemost 1991;65:425-431.

4 Carmeliet P, Moons L, Stassen J-M, et al. Vascular wound healing and neointima formation induced by perivascular electric injury in mice. Am J Pathol 1997;150:761-776.

5 Farrehi PM, Ozaki CK, Carmeliet P, et al. Regulation of arterial thrombolysis by plasminogen activator inhibitor-1 in mice. Circulation 1998;97:1002-1008.

6 Roesken F, Ruecker M, Vollmar B, et al. A new model for quantitative in vivo microscopic analysis of thrombus formation and vascular recanalisation: the ear of the hairless $(\mathrm{hr} / \mathrm{hr})$ mouse. Thromb Haemost 1997;78:1408-1414.

7 Rosenblum WI, Nelson GH, Povlishock JT. Laserinduced endothelial damage inhibits endotheliumdependent relaxation in the cerebral microcirculation of the mouse. Circ Res 1987;60:169-176.

8 Rosen ED, Raymond S, Zollman A, et al. Laser-induced noninvasive vascular injury models in mice generate platelet- and coagulation-dependent thrombi. Am J Pathol 2001;158:1613-1622.

9 Booth RF, Martin JF, Honey AC, et al. Rapid development of atherosclerotic lesions in the rabbit carotid artery induced by perivascular manipulation. Atherosclerosis 1989;76:257-268.

10 Kumar A, Lindner V. Remodeling with neointima formation in the mouse carotid artery after cessation of blood flow. Arterioscler Thromb Vasc Biol 1997; 17:2238-2244.

11 Kuzuya M, Kanda S, Sasaki T, et al. Deficiency of gelatinase a suppresses smooth muscle cell invasion and development of experimental intimal hyperplasia. Circulation 2003;108:1375-1381.

12 von der Thusen JH, van Berkel TJ, Biessen EA. Induction of rapid atherogenesis by perivascular carotid collar placement in apolipoprotein E-deficient and low-density lipoprotein receptor-deficient mice. Circulation 2001;103:1164-1170.

13 Alroy J, Goyal V, Skutelsky E. Lectin histochemistry of mammalian endothelium. Histochemistry 1987;68: 603-607.

14 Mattsson G, Carlsson PO, Olausson K, et al. Histological markers for endothelial cells in endogenous and transplanted rodent pancreatic islets. Pancreatology 2002;2:155-162.

15 Kurz KD, Main BW, Sandusky GE. Rat model of arterial thrombosis induced by ferric chloride. Thromb Res 1990;60:269-280.

16 Kawasaki T, Dewerchin M, Lijnen HR, et al. Mouse carotid artery ligation induces platelet-leukocytedependent luminal fibrin, required for neointima development. Circ Res 2001;88:159-166.

17 Hagihara H, Nomoto A, Mutoh S, et al. Role of inflammatory responses in initiation of atherosclerosis: effects of anti-inflammatory drugs on cuff-induced leukocyte accumulation and intimal thickening of rabbit carotid artery. Atherosclerosis 1991;91:107-116.

18 Suffredini AF, Harpel PC, Parrillo JE. Promotion and subsequent inhibition of plasminogen activation after administration of intravenous endotoxin to normal subjects. N Engl J Med 1989;320:1165-1172.

19 Wagner DD. Cell biology of von Willebrand factor. Annu Rev Cell Biol 1990;6:217-246.

20 Blann AD, McCollum CN. von Willebrand factor, endothelial cell damage and atherosclerosis. Eur J Vasc Surg 1994;8:10-15.

21 Lip GY, Blann A. von Willebrand factor: a marker of endothelial dysfunction in vascular disorders? Cardiovasc Res 1997;34:255-265.

22 Mannucci PM. von Willebrand factor: a marker of endothelial damage? Arterioscler Thromb Vasc Biol 1998;18:1359-1362.

23 de Groot PG, Sixma JJ. Role of von Willebrand factor in the vessel wall. Semin Thromb Hemost 1987;13: 416-424.

24 McRitchie DI, Girotti MJ, Glynn MF, et al. Effect of systemic fibrinogen depletion on intraabdominal abscess formation. J Lab Clin Med 1991;118:48-55.

25 Hara H, Kitajima A, Shimada H, et al. Antithrombotic effect of MCI-9042, a new antiplatelet agent on experimental thrombosis models. Thromb Haemost 1991;66:484-488.

26 Roque M, Fallon JT, Badimon JJ, et al. Mouse model of femoral artery denudation injury associated with the rapid accumulation of adhesion molecules on the luminal surface and recruitment of neutrophils. Arterioscler Thromb Vasc Biol 2000;20:335-342. 